\title{
Implementación de un programa de reciclaje en pabellón
}

\section{Performance of a recycling program in the operating room}

\author{
Víctor Contreras I. ${ }^{1,3,4}$, Ricardo Fuentes H. ${ }^{1,5,6}$, Constanza Barros P. 1,7,8, Osman Casanueva F. ${ }^{1,9}$, \\ Gabriela Barrueto D. ${ }^{1,8}$
}

\begin{abstract}
Introduction: The waste management of a hospital center affects people both those who receive health care and the environment that receives them. The impact on the environment of the task of peri-operative care has not yet been properly studied. It is known that of the 105 tons of garbage monthly from the health center understudy go to the landfills of the municipality. However, there are different elements, such as low-density plastics (LDP), which can be recycled. Objective: implement a clean low-density plastics recycling program in the central Operating Room of the UC-Christus Clinical Hospital. Methodology: In this report, a prospective longitudinal study was conducted with a sample for convenience, in which an intervention was scheduled in series repeated over time from 2017 to 2018. Residents of anesthesia, anesthesiologists, nurses, nurse technicians, and hospital support personnel participated. Results: In total 200 employees participated. During the pilot phase, $30 \mathrm{~kg}$ of plastics were quantified, later increasing to $100 \mathrm{~kg}$ of LDP in the subsequent phases. It began with the transfer phase to other pavilions within the healthcare center and to other centers in the metropolitan region. Finally, an alternative was started with the municipalities. Conclusions: It was possible to quantify the amount of clean LPD for recycling. It was possible to involve the collaborators of the institution. This is the first program reported that involves an intervention for LPD.
\end{abstract}

\section{Key words:}

Recycling, operating room, anesthesia, health planning

\footnotetext{
Pontificia Universidad Católica de Chile.

Facultad de Medicina.

Magíster en Enfermería, Enfermero.

División de Anestesiología.

MD Anestesiólogo

Red de Salud UC-Christus.

Magíster en Administración en Salud.

Enfermera.

Ingeniero de Ejecución en Administración y Finanzas.
}

Fecha de recepción: 18 de enero de 2019

Fecha de aceptación: 29 de enero de 2019

\section{ORCID}

https://orcid.org/0000-0002-6081-0430

Correspondencia:

Víctor Contreras

División de Anestesiología.

Email: vecontre@uc.cl 


\section{RESUMEN}

Introducción: El manejo de los desechos de un centro hospitalario afecta a las personas tanto a los que reciben atención de salud como también al medioambiente que recibe éstos. El impacto en el ambiente del quehacer de la atención perioperatoria aún no ha sido estudiado apropiadamente. Se sabe que 105 toneladas de basura mensuales del centro asistencial en estudio van a los vertederos de la municipalidad. Sin embargo, existen distintos elementos, como los plásticos de baja densidad (PBD), limpios los cuales pueden ser reciclados. Objetivo: Implementar de un programa de reciclaje de plásticos de baja de densidad limpios en el Servicio de Pabellón Central del Hospital Clínico UC-Christus. Material y Métodos: En este reporte se realizó un estudio prospectivo longitudinal con una muestra por conveniencia, en la cual se programó una intervención en series repetidas en el tiempo durante 2017 al 2018. Participaron residentes de anestesia, anestesiólogos, enfermeras, TENS, personal de apoyo del hospital entre otros. Resultados: En total 200 colaboradores participaron. Durante la fase piloto se cuantificó $30 \mathrm{~kg}$ de plásticos, posteriormente, se incrementó a $100 \mathrm{~kg}$ de PBD en las fases posteriores. Se comenzó con la fase de transferencia hacia otros pabellones dentro del centro asistencial y hacia otros centros de la Región Metropolitana. Finalmente, se comenzó a buscar alternativa con las municipalidades. Conclusiones: Se logró cuantificar la cantidad de PBD limpios para reciclar. Se logró involucrar a los colaboradores de la institución. Este es el primer programa reportado que involucra una intervención para el PBD.

\section{Palabras clave:}

Reciclaje, sala de operaciones, anestesia, planificación en salud

\section{Introducción}

Lis

a Organización Mundial de la Salud (OMS) ha estimado que un $85 \%$ de los residuos generados por los hospitales pueden ser clasificados como comunes no peligrosos, semejantes a la basura doméstica, siendo el $15 \%$ restante se considerado como material peligroso que puede ser infeccioso, tóxico o radioactivo y que necesita ser tratado antes de eliminarse[1],[2].

Con el procesamiento de ambos residuos se podría estimar la huella de carbono de un hospital, es decir, cuánto de estos residuos afectarían sobre el cambio climático, más allá de los grandes emisores de dióxido de carbono[3].

Por otro lado, existen materiales reutilizables y otros tratables para ser reciclados dentro de los hospitales. Así mismo, existe una iniciativa mundial de la OMS de prevenir las enfermedades a través de ambientes saludables, en la cual se han propuesto y se han desarrollado varios incentivos entorno al cuidado del medioambiente, los cuales aún no se traducen en programas concretos locales y/o políticas para prevenir el daño al medio ambiente en temas de reciclaje hospitalario[4].

Así también, la incineración de residuos médicos (proceso de tratamiento de la mayoría de los residuos peligrosos y basuras complejas), genera diversos gases y compuestos peligrosos, entre ellos ácido clorhídrico, dioxinas, furanos y metales tóxicos; como el plomo, cadmio y mercurio contaminantes[5],[6].

Cabe contar también que los gases clínicos usados en los hospitales y la incineración de residuos sólidos producen emisiones de gases de efecto invernadero, incluido el metano y los halogenados, gases que son hasta 20 veces más contaminantes y que quedan por años en la atmósfera, tema que tampoco está incluido en la palestra de asuntos medioambientales actuales.

En nuestro país los hospitales tienen regulaciones claras acerca de la generación y tratamiento de la basura compleja y de alto impacto al ambiente (6). Cerca de 120 toneladas de basura se eliminan cada mes desde nuestro centro hospitalario central que cuenta con 450 camas (considerando 2.300 egresos mensuales y una estadía promedio de 5,2 días por paciente).

Entre el $10 \%$ al $15 \%$ de la basura hospitalaria es de alto costo, es decir, 18 toneladas mensuales necesita ser tratada antes de ser eliminada. Considerando que, el costo en nuestro país de procesar la basura compleja hospitalaria es de aproximadamente $\$ 1.300$ por kilo. 
De las basuras del hospital reciclables cerca de 100 kilos de plásticos de baja densidad -PBD- (similar a las bolsas plásticas de supermercado) llegan a limpias y no tocan al paciente, por lo cual pueden ser enviadas a reciclar dentro de Hospital Clínico UC Christus.

Dado lo anterior, nuestro Hospital, en alianza con la Dirección de Sustentabilidad de la Pontificia Universidad Católica de Chile y dirigida por la División de Anestesiología de dicha casa de estudio, se propuso implementar un programa de reciclaje de plásticos de baja densidad limpios del pabellón central de nuestro centro de atención.

\section{Materiales y Métodos}

En este reporte se realizó un estudio prospectivo longitudinal con una muestra por conveniencia en la cual se programó una intervención en series repetidas en el tiempo durante junio de 2017 a diciembre de 2018.

Las etapas del proyecto fueron: 2017 evaluar el o los materiales posibles de reciclar dentro del ambiente de pabellón central, capacitar a los colaboradores (enfermeras, técnicos de enfermería, anestesiólogos, residentes de anestesiología, personal de apoyo, personal de la empresa HHS, la dirección de Servicios Generales, la Gerencia General del Hospital y la Gerencia de Misión de la Red Salud UC-Christus) en el programa de hospital verde y saludable.

La segunda etapa fue cuantificar mensualmente el plástico de baja densidad posible de reciclar, determinar el lugar de acopio dentro del centro y el destino final del reciclador de plásticos. Se evaluaron más de 10 empresas que declaraban en sus páginas web la posibilidad de hacer el proceso de reciclaje y entregar un certificado del producto final.

La tercera etapa fue incluir a otros actores dentro del pabellón como son el Almacén Quirúrgico del pabellón central, realizar las capacitaciones continuas (mensualmente) a los colaboradores, elección de "capitanes dentro del pabellón", creación de un grupo WhatsApp para comunicación rápida y propuesta de meta para lograr incentivar a los colaboradores del programa.

La cuarta etapa, fue mejorar el sistema de segregación de PBD, replantear las metas e incentivos, integrar a nuevos servicios, participar de concursos internos y externos de financiamiento del programa, extender el programa a la Clínica San Carlos de Apoquindo, creación de las Islas de reciclaje para las áreas comunes de familiares y buscar alternativas con las municipalidades de Santiago y Las Condes para optimizar el proceso de retiro y reciclado.

\section{Resultados}

\section{Equipo de colaboradores}

Dentro de las principales tareas de este programa fue organizar el equipo humano, donde los coordinadores del programa: Víctor Contreras, enfermero de la División de Anestesiología, Gabriela Barrueto, enfermera del Pabellón Central, Ricardo Fuentes, jefe del Pabellón Central, Osman Casanueva, jefe de Servicios Generales del Hospital Clínico UC-Christus y Constanza Barros, enfermera jefa del Almacén Quirúrgico del Pabellón $6^{\text {to }}$, lograron participar de una visita de aprendizaje mutuo y colaboración en el hospital Sick Kids, la organización UHN y la coalición Green Health Care Canadá, en Toronto. Este premio fue logrado a la participación del concurso de Innovación UC Sustentable 2018, donde el proyecto logró el primer lugar.

Se logró generar incentivos asociados a las metas para los colaboradores y capitanes recicladores: Marlene Fuentes, enfermera jefe Almacén Quirúrgico, Javiera González, Graciela Díaz, Álvaro Aguilar y Pamela Matus todas enfermeras del pabellón central, más Marta Pinochet, Roxana Quezada y Miriam Rebolledo técnicos de enfermería del pabellón central. Dichos incentivos fueron un desayuno para todo el equipo de pabellón central y personal de apoyo HHS cuya meta fue 30 kilos de PBD, limpios, secos y sin basuras no reciclables.

\section{Plásticos de baja densidad e islas de reciclaje}

Posteriormente, se propuso lograr 100 kilos de PBD, limpios, secos y sin basuras no reciclables, sin embargo, a diciembre de 2018 aún esta meta no ha sido lograda.

El promedio mensual del programa de eliminación adecuado de PBD en el servicio de pabellón central del hospital Clínico UC-Christus (Tabla 1).

En noviembre de 2018 comenzó con el reciclaje de PBD en el pabellón del 6to piso. Proceso que se inicia cuantificando la cantidad total mensual de PBD a reciclar correctamente durante tres meses. Con esto se podrá establecer una meta para marzo de 2019.

En la actualidad, tanto en el Hospital Clínico UCChristus y la Clínica UC, se cuenta con 6 islas de reciclaje en las áreas comunes. Distribuidas en el piso $7^{\mathrm{mo}}$, $6^{\text {to }}, 3^{\text {ro }}, 1^{\text {ro }}, 1^{\text {er }}$ subterráneo y $1^{\text {er }}$ piso Clínica UC, en 


\begin{tabular}{lc}
\multicolumn{2}{c}{$\begin{array}{c}\text { Tabla 1. Resumen del reciclaje de plásticos del pabe- } \\
\text { llón central }\end{array}$} \\
\hline Trimestre & PBD* Kg \\
Oct - dic 2017 & 205 \\
Ene - mar 2018 & 118 \\
Abr - jun 2018 & 332 \\
Jul - sep 2018 & 294 \\
Oct - dic 2018 & 258,5 \\
Total & $1.207,5$ \\
\hline
\end{tabular}

los cuales se segregan botellas plásticas, envases de vidrio, latas y pilas (Tabla 2).

La Clínica San Carlos de Apoquindo quedó con la tarea de iniciar este proceso de segregación, principalmente, por la cantidad de botellas plásticas que se eliminan del centro.

\section{Actores municipales}

Durante noviembre y diciembre de 2018 se pudo concretar una reunión con la oficina de medioambiente, en el cual se expuso la necesidad de contar con un centro de tratamiento de PBD en la comuna. En dicha reunión la I. Municipalidad de Santiago nos informó que es una tarea que conlleva una nueva redistribución de acciones en las cuales están involucradas el transporte de los materiales reciclados y el centro de acopio que está en planes de construcción en el Parque de los Reyes.

Con respecto a la Clínica San Carlos de Apoquindo están comenzando con la exploración del material icónico para iniciar el reciclaje, con el fin de montar un piloto dentro de sus instalaciones.

La I. Municipalidad de Las Condes, no respondió a los correos electrónicos enviados al director de aseo y ornato de dicho municipio, por lo cual no se ha avanzado en el destino final de los residuos de la Clínica San Carlos de Apoquindo.

\section{Discusión y desafíos futuros}

En la actualidad el Ministerio de Medio Ambiente contempla en su plan de desarrollo al menos 4 estra- tegias relacionadas con los residuos, una es la consulta ciudadana para el Reglamento de la ley de fomento del reciclaje, un registro de entidades sin fines de lucro chilenas que contribuyen en la gestión de residuos, un fondo concursable para el reciclaje del año 2019 para municipalidades y asociaciones municipales con el foco en avanzar en la implementación de la Ley $N^{\circ}$ 20.920. Así también, cuenta con un Sistema Globalmente Armonizado de clasificación y etiquetado de productos químicos (GHS por sus siglas en inglés) cuya iniciativa mundial es promover criterios uniformes para la clasificación y etiquetado de los productos químicos que servirá para definir y comunicar los peligros físicos, para la salud y para el medio ambiente.

La armonización con el Ministerio de Salud[7] al parecer no es del todo conjunta, dado que el Reglamento sobre Manejo de Residuos de Establecimientos de Atención de Salud[8] está operado y supervisado por dicho ministerio a través de las SEREMIS, sin embargo, aún no está aunada a las políticas relacionadas con el reciclaje de residuos y/o incentivos para los centros de atención que adoptan políticas pro medioambientales.

Dado esto, el programa de reciclaje de nuestro centro se ve limitado y estamos siendo apoyados por la Dirección de Sustentabilidad de la UC, quienes nos facilitan sus instalaciones en el campus San Joaquín y Casa Central para enviar nuestros residuos reciclables.

Sumarse a las iniciativas internacionales de reporte de residuos y uso energético adecuado es una de las alternativas para mejorar nuestros procesos y colaborar con la salud pública. Además, conlleva el ahorro de dinero; así por ejemplo encontramos con el formulario de la coalición Green Health Care en Canadá[10], el cual nos dio la oportunidad de reportar nuestras metas y ser parte de este grupo de centros que tienen políticas concretas en términos ecológi$\cos [9],[10]$.

Siguiendo lo promovido por la OMS, iniciativa llamada "la salud en la economía verde", se espera poder reunir a los diferentes hospitales y centros de atención del país para buscar alternativas conjuntas cuyo foco sea reducir la huella de carbono del sector de la salud[4].

La normativa REAS propone el incentivo de una política de compras que asegure la selección de mé-

\begin{tabular}{lcccc}
\multicolumn{5}{c}{ Tabla 2. Resumen de la cantidad de elementos segregados } \\
\hline Período & Plásticos kg & Vidrios kg & Latas kg & Pilas kg \\
Jun-nov 2018 & 148,75 & 213,55 & 62,47 & 146,81 \\
\hline
\end{tabular}


todos o insumos que desperdicien menos recursos o que generen menos residuos[6]. Sin embargo, aún estas declaraciones no se vuelven del todo operativa tanto para los prestadores de salud públicos como privados.

Existen empresas privadas destinadas a trabajar en este proceso de basuras complejas, no obstante, el costo es asumido aún por los centros de atención que generan la basura.

Realizar las mediciones acerca de la liberación de halogenados al ambiente intra-pabellón y al medioambiente, es una tarea pendiente y que como centro estamos trabajando, dado que los gases anestésicos pueden provocar problemas en la salud de los funcionarios de las zonas quirúrgicas, los cuales deberían ser reportados a los organismos encargados de la salud laboral y seguridad social. La ausencia de sistemas de extracción de gases adecuados dentro de los hospitales, así como el inadecuado control de las fugas por mantenimiento de las instalaciones, la mala ventilación de las salas, los flujos de gases frescos excesivo y la despreocupación y desinterés del equipo de salud, son el problema de contaminación.
En nuestro país existe el Registro de Emisiones y Transferencias de Contaminantes[5], en el cual se especifican la base de datos que contiene información periódica y actualizada sobre sustancias químicas contaminantes o potencialmente dañinas para la salud y el medioambiente, que son emitidas directamente al suelo, al aire o al agua por establecimientos industriales $u$ otras actividades como el transporte $o$ la agricultura. Además, incluye información sobre el tratamiento o eliminación de residuos peligrosos[5], no obstante, no aparecen incluidos los hospitales.

\section{Conclusiones}

Se logró cuantificar la cantidad de PBD limpios para reciclar. Se logró involucrar a los colaboradores de la institución. Este es el primer programa reportado que involucra una intervención dentro de un centro asistencial para el manejo de PBD en pabellón. Pendiente quedan los desafíos con las políticas públicas del MINSAL y del Ministerio de Medioambiente en el desarrollo en pro de la protección del mundo en que vivimos.

\section{Referencias}

1. World Wide Fund For Nature. Living Report Planet 2016. https://www.who.int/sustainable-development/health-sector/ health-risks/solid-waste/en/

2. WHO. Health-care waste management. Health and Sustainable Development https://www.who. int/sustainable-development/ health-sector/health-risks/solidwaste/en/

3. Espíndola C, Valderrama JO Huella del Carbono. Parte 1: Conceptos, Métodos de Estimación y Complejidades Metodológicas. Inf Tecnol. 2012;23(1):163-76. https:// doi.org/10.4067/S071807642012000100017
4. WHO. Health in the Green Economy 2014. Acceso 02 de diciembre de 2018. Disponible en: https://www.who.int/hia/ green_economy/en/

5. Ministerio del Medioambiente. Reporte 2005-2009 del Registro de emisiones y transferencias de contaminantes, RETC2011. Acceso 05 de diciembre de 2018. Disponible en: http://www.retc. cl/wp-content/uploads/2015/07/ articles-56962_Quinto_reporte_RETC.pdf

6. Instituto de Salud Pública. Manejo de residuos de establecimientos de atención de salud 2010. Acceso 05 de diciembre de 2018. Disponible en: http:// www.ispch.cl/sites/default/files/ manual\%20reas.pdf
7. Ministerio del Medioambiente. Oficina Residuos. Acceso 05 de diciembre de 2018. Disponible en: http://portal.mma.gob.cl/ residuos/

8. Red de Hopsitales Verdes y Saludables. Trabajando Juntos por la Salud Ambiental 2010. Acceso 05 de diciembre de 2018. Disponible en: http://www.hospitalesporlasaludambiental.net/

9. Sustainable Development Unit. Route Map 2013. Acceso 12 de diciembre de 2018. Disponible en: https://www.sduhealth.org. uk/policy-strategy/route-map.aspx

10. Care GH. Who We Are. Canadian Coalition for Green Health Care 2012. Acceso 12 de diciembre de 2018. Disponible en: http://greenhealthcare.ca/ 